\title{
High-frequency Graviton from Inflaton Oscillation
}

\author{
Yohei Ema ${ }^{(a)}$, Ryusuke Jinno ${ }^{(a)}$ and Kazunori Nakayama ${ }^{(b, c)}$ \\ (a) DESY, Notkestrabe 85, D-22607 Hamburg, Germany \\ (b) Department of Physics, Faculty of Science, \\ The University of Tokyo, Bunkyo-ku, Tokyo 113-0033, Japan \\ (c) Kavli IPMU (WPI), The University of Tokyo, Kashiwa, Chiba 27r-8583, Japan
}

\begin{abstract}
We point out that there is a high-frequency tail of the stochastic inflationary gravitational wave background that scales as $f^{-1 / 2}$ with frequency $f$. This contribution comes from the graviton vacuum fluctuation amplified by the inflaton coherent oscillation during the reheating stage. It contains information on inflaton properties such as the inflaton mass as well as the thermal history of the early Universe.
\end{abstract}




\section{Contents}

1 Introduction $\quad 1$

2 Graviton in inflationary universe 2

3 High frequency graviton production 3

4 Stochastic gravitational wave background revisited 6

5 Discussion $\quad 7$

\section{Introduction}

Gravitational wave (GW) provides us with a new way to probe our universe. It interacts only very weakly with matters, and hence preserves the information on source objects imprinted in its spectrum during its propagation. In particular, $\mathrm{GW}$ is a unique way to probe the early universe, as all the other messengers (such as photons and neutrinos) interact strongly with matters and hence lost their information in the (sufficiently) early universe. For instance, inflation generically predicts GWs that are excited during the quasi de Sitter phase [1-3]. They are one of the main targets of the modern cosmological observatories since they provide us with the information on the inflationary energy scale. GWs are also expected to be produced during the course of thermal history after inflation, such as preheating [4], cosmological defects [5], and phase transitions [6,7], although the existence of these contributions is more model-dependent (see Ref. [8] for a recent review).

In this paper, we point out the existence of yet another source of GW. In general, an inflaton starts to oscillate around the bottom of its potential after inflation, before eventually decaying into other particles and hence completing the reheating. This coherent oscillation of the inflaton during the inflaton oscillation epoch produces GWs through gravitational interaction, which can be interpreted as inflaton annihilation into gravitons mediated by gravity itself [9]. This contribution extends toward the high frequency region beyond the inflationary GWs. Since it is produced from the inflaton oscillation, the GW spectrum contains a variety of information on the inflaton sector, such as the inflaton mass scale and more generally the shape of the inflaton potential around its bottom. It also depends on the inflaton decay rate and hence the reheating temperature.

It is quite challenging to detect this contribution with the current and near-future GW detectors [10-16] (see also Refs. [17-21] for ideas for high-frequency graviton detection), not only because of its overall normalization but also because of its high characteristic frequency. Furthermore, there are other GW sources in the high frequency region that we expect to be present in general, such as the contribution from the standard model (SM) thermal plasma [22,23], the bremsstrahlung from the inflaton decay $[24,25]$ as well as those from preheating [4]. These other contributions can hide our GWs, depending on the model 
parameters and the frequency. Nevertheless, we think it meaningful to point out the existence of the GWs produced during the inflaton oscillation epoch, as this contribution imprints quite interesting information on the inflaton sector that is usually hard to reach. We hope that a development of GW detection technology eventually enables us to probe the high frequency region such that we can gain information on the inflaton sector in the future.

This paper is organized as follows. In Sec. 2, we review the equation of motion of the graviton and the graviton production during inflation. Sec. 3 is the main part of this paper, where we compute the GW production from the inflaton oscillation after inflation both analytically and numerically. In Sec. 4, we show the resultant GW spectrum, especially its dependence on the model parameters such as the inflaton mass and the reheating temperature. Sec. 5 is devoted to the discussion, where we compare our contribution with the contributions from the SM thermal plasma and the bremsstrahlung from the inflaton decay.

\section{Graviton in inflationary universe}

We consider the Einstein-Hilbert action plus the inflaton action as

$$
S=\int d t d^{3} x \sqrt{-g}\left(\frac{M_{P}^{2}}{2} R+\mathcal{L}_{\phi}\right)
$$

The metric is expanded as

$$
d s^{2}=-d t^{2}+a^{2}(t)\left(\delta_{i j}+h_{i j}\right) d x^{i} d x^{j}=a^{2}(t)\left[-d \tau^{2}+\left(\delta_{i j}+h_{i j}\right) d x^{i} d x^{j}\right],
$$

where we have taken the transverse-traceless gauge: $h_{i}^{i}=\partial^{i} h_{i j}=0$. The graviton action is given by

$$
\begin{aligned}
S & =\int d t d^{3} x a^{3} \frac{M_{P}^{2}}{8}\left[\left(\dot{h}_{i j}\right)^{2}-\frac{1}{a^{2}}\left(\partial_{l} h_{i j}\right)^{2}\right] \\
& =\sum_{\lambda=+, \times} \int \frac{d \tau d^{3} k}{(2 \pi)^{3}} \frac{1}{2}\left[\left|h_{\lambda}^{\prime}(k)\right|^{2}-\omega_{k}^{2}\left|h_{\lambda}(k)\right|^{2}\right], \quad \omega_{k}^{2} \equiv k^{2}-\frac{a^{2} R}{6}
\end{aligned}
$$

where the prime denotes the derivative with respect to the conformal time $\tau$, and $\lambda=+, \times$ denotes the two polarization states of the graviton. We have defined the canonical graviton in the momentum space as

$$
\frac{a M_{P}}{2} h_{i j}(t, \vec{x})=\sum_{\lambda=+, \times} \int \frac{d^{3} k}{(2 \pi)^{3}} h_{\lambda}(\vec{k}, \tau) e^{i \vec{k} \cdot \vec{x}} \epsilon_{i j}^{\lambda},
$$

where $\epsilon_{i j}^{\lambda}$ denotes the polarization tensor, which satisfies $\epsilon_{i j}^{\lambda} \epsilon_{i j}^{\lambda^{\prime}}=\delta_{\lambda \lambda^{\prime}}$. The free graviton action (3) is the same as the minimally-coupled massless scalar field. Thus gravitational production of gravitons during the inflation and reheating era is treated in the same way as the minimal scalar field which is extensively studied in Refs. [9, 26-28]. 
Let us introduce a creation and annihilation operator for the graviton:

$$
h_{\lambda}(\vec{k}, \tau)=\widetilde{h}_{\lambda}(\vec{k}, \tau) a_{\lambda, \vec{k}}+\widetilde{h}_{\lambda}^{*}(\vec{k}, \tau) a_{\lambda,-\vec{k}}^{\dagger},
$$

where they satisfy the commutation relation $\left[a_{\lambda, \vec{k}}, a_{\lambda^{\prime}, \vec{k}^{\prime}}^{\dagger}\right]=(2 \pi)^{3} \delta\left(\vec{k}-\vec{k}^{\prime}\right) \delta_{\lambda \lambda^{\prime}}$. The equation of motion is given by

$$
\widetilde{h}_{\lambda}^{\prime \prime}(k)+\omega_{k}^{2} \widetilde{h}_{\lambda}(k)=0 .
$$

The solution to the equation of motion and its approximate form in the high and low frequency limit, which satisfies the Bunch-Davies boundary condition, during inflation is given by

$$
\widetilde{h}_{\lambda}(k, \tau)=-\frac{1}{\sqrt{2 k}} \sqrt{\frac{-\pi k \tau}{2}} H_{3 / 2}^{(1)}(-k \tau) \simeq\left\{\begin{array}{ll}
\frac{1}{\sqrt{2 k}} e^{-i k \tau} & \text { for }-k \tau \gg 1 \\
i \frac{a H_{\text {inf }}}{\sqrt{2} k^{3 / 2}} & \text { for }-k \tau \ll 1
\end{array},\right.
$$

where $H_{3 / 2}^{(1)}(x)$ denotes the Hankel function of the first kind and we used $\tau=-\left(a H_{\text {inf }}\right)^{-1}$ during inflation with $H_{\text {inf }}$ being the inflationary Hubble scale. It is well known that the superhorizon modes $\left(-k \tau_{\text {end }} \lesssim 1\right.$ where the subscript "end" represents the end of inflation) have (nearly) scale invariant power spectrum: ${ }^{\# 1}$

$$
\mathcal{P}_{h}\left(k, \tau_{\text {end }}\right) \equiv \frac{k^{3}}{\pi^{2} a^{2}}\left|\widetilde{h}_{\lambda}(k)\right|^{2}=\frac{H_{\text {inf }}^{2}}{2 \pi^{2}} \quad \text { for } \quad-k \tau_{\text {end }} \ll 1 .
$$

On the other hand, shorter wavelength modes $\left(-k \tau_{\text {end }} \gtrsim 1\right)$ never exit the horizon. However, it does not mean that shorter wavelength modes are not excited. Below we will evaluate the production of these short wavelength graviton modes during the inflaton oscillation epoch after inflation.

\section{$3 \quad$ High frequency graviton production}

Let us consider the high-frequency modes that never exit the horizon: $-k \tau_{\text {end }} \gtrsim 1$. After inflation ends, the inflaton coherent oscillation begins and the graviton wave function is modified through the (rapidly-oscillating) $a^{2} R$ term in the equation of motion. In this case it is convenient to parameterize the wave function in terms of the Bogoliubov coefficients $\alpha_{k}, \beta_{k}$ as

$$
\widetilde{h}_{\lambda}(k, \tau)=\alpha_{k}(\tau) v_{k}(\tau)+\beta_{k}(\tau) v_{k}^{*}(\tau)
$$

\#1 Often the graviton power spectrum is defined by the original basis before the canonical rescaling. In such a case the graviton power spectrum is given by $\overline{\mathcal{P}}_{h}(k) \equiv\left(2 / M_{P}\right)^{2} \mathcal{P}_{h}(k)=2 H_{\text {inf }}^{2} /\left(\pi M_{P}\right)^{2}$ and the tensor-to-scalar ratio is defined as $r=\overline{\mathcal{P}}_{h}(k) / \mathcal{P}_{\zeta}(k)$ with $\mathcal{P}_{\zeta}$ being the power spectrum of the curvature perturbation. 
where

$$
v_{k}(\tau)=e^{-i \Omega_{k}(\tau)}, \quad \Omega_{k}(\tau) \equiv \int \omega_{k} d \tau
$$

The equation of motion is rewritten as

$$
\alpha_{k}^{\prime}(\tau)=\frac{\omega_{k}^{\prime}}{2 \omega_{k}} \beta_{k}(\tau) e^{2 i \Omega_{k}}, \quad \beta_{k}^{\prime}(\tau)=\frac{\omega_{k}^{\prime}}{2 \omega_{k}} \alpha_{k}(\tau) e^{-2 i \Omega_{k}}
$$

They satisfy the normalization condition $\left|\alpha_{k}(\tau)\right|^{2}-\left|\beta_{k}(\tau)\right|^{2}=1$. The initial condition is $\alpha_{k}=1$ and $\beta_{k}=0$ for $-k \tau \rightarrow \infty$. The renormalized graviton energy density is expressed as

$$
a^{4}(\tau) \rho_{h}(\tau)=2 \int \frac{d^{3} k}{(2 \pi)^{3}} \omega_{k}\left|\beta_{k}(\tau)\right|^{2} .
$$

We define the graviton energy spectrum as

$$
\rho_{h}(\tau)=\int \rho_{h, k}(\tau) d \ln k, \quad a^{4}(\tau) \rho_{h, k}(\tau)=\frac{k^{3} \omega_{k}}{\pi^{2}}\left|\beta_{k}(\tau)\right|^{2} .
$$

Thus it is sufficient to evaluate $\beta_{k}(\tau)$ to obtain the graviton energy spectrum. Numerically, one can integrate the equation (11) to obtain $\beta_{k}(\tau)$ given an inflation model.

Fig. 1 shows the result of our numerical calculation. We assumed a chaotic inflation model with a quadratic potential $V=m_{\phi}^{2} \phi^{2} / 2$ for concreteness, ${ }^{\# 2}$ and solved the following equations

$$
\begin{aligned}
& 3 M_{P}^{2} \mathcal{H}^{2}=a^{2}\left(\rho_{\phi}+\rho_{r}\right), \\
& \phi^{\prime \prime}+\left(2 \mathcal{H}+a \Gamma_{\phi}\right) \phi^{\prime}+a^{2} \frac{d V}{d \phi}=0 \\
& \rho_{r}^{\prime}+4 \mathcal{H} \rho_{r}=a \Gamma_{\phi} \rho_{\phi},
\end{aligned}
$$

where $\rho_{\phi}=\phi^{\prime 2} / 2 a^{2}+V$ is the inflaton energy density, $\rho_{r}$ is the radiation energy density, $\Gamma_{\phi}$ is the inflaton total decay width and $\mathcal{H}=a^{\prime} / a$ denotes the conformal Hubble scale. Eq. (11) is solved numerically with this background. The inflaton decay rate is (hypothetically) taken to be zero in the left panel and the spectrum is evaluated during the inflaton domination. In the right panel the inflaton decay rate is taken to be $\Gamma_{\phi}=\left(10^{-1}, 10^{-2}, 10^{-3}\right) \times m_{\phi}$ and the spectrum is evaluated during the radiation domination. One can see that the graviton energy spectrum shows $k^{-1 / 2}$ behavior as expected. Below we compare this result with an analytic estimate.

In order to evaluate the graviton energy spectrum, it is convenient to interpret the graviton production during the reheating era as the inflaton annihilation into a graviton pair, as

\footnotetext{
\#2 The chaotic inflation [29] with a quadratic potential is now disfavored by the cosmological observation, but a slight modification on the potential makes the model viable [30,31]. Since we are mainly interested in the inflaton oscillation regime, such a modification is irrelevant for the discussion below.
} 

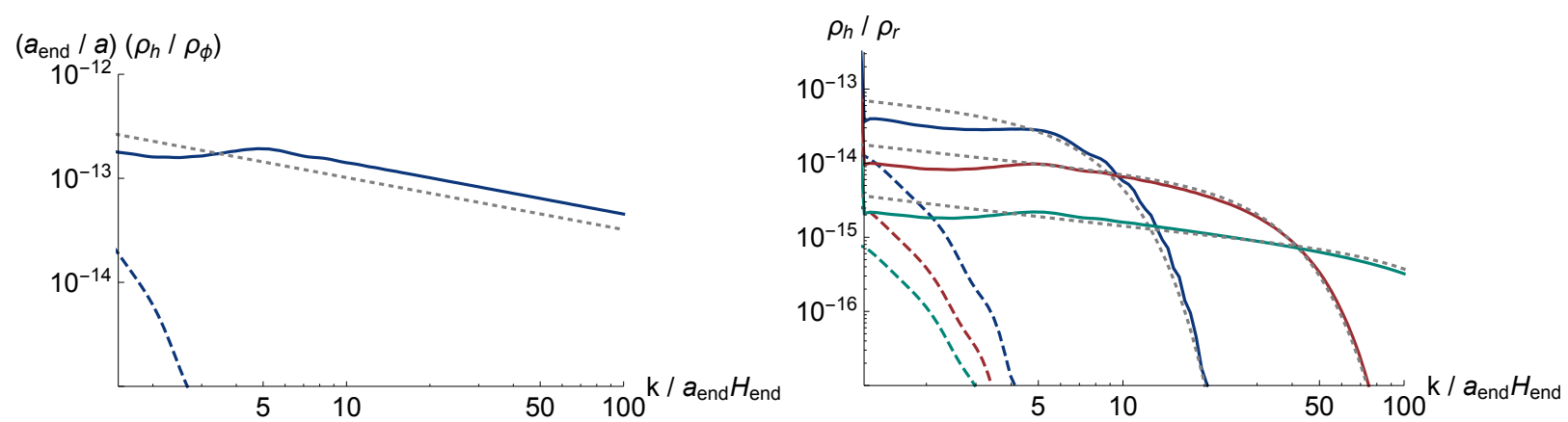

Figure 1: Graviton energy spectrum after inflation. The numerical solutions of Eq. (11) with Eqs. (14)-(16) are shown as the solid lines, while the analytic estimates, i.e. Eq. (19), are shown as the gray-dotted line, for the quadratic inflaton potential $V=m_{\phi}^{2} \phi^{2} / 2$. For comparison, the numerical results without the inflaton oscillation (Eq. (20)) are shown as the dashed line. The modes shown here never exit the horizon. (Left) The inflaton decay rate is taken to vanish, and the spectrum is evaluated during the inflaton domination. The ratio $\rho_{h} / \rho_{\phi}$ is multiplied with the ratio of the scale factor at the inflation end and at the evaluation time to cancel out the dependence on the evaluation time. (Right) The inflaton decay rate is taken to be $\Gamma_{\phi}=10^{-1} m_{\phi}$ (blue), $10^{-2} m_{\phi}$ (red) and $10^{-3} m_{\phi}$ (green), and the spectrum is evaluated during the radiation domination.

emphasized in Refs. [9,26,27]. Taking account of two polarization states of the graviton, the effective inflaton annihilation rate is estimated as $\# 3$

$$
\Gamma^{\text {(grav) }}(\phi \phi \rightarrow h h) \simeq \frac{1}{192 \pi} \frac{\rho_{\phi} m_{\phi}}{M_{P}^{4}}
$$

Each annihilation produces a pair of gravitons with energy $m_{\phi}$ that are then redshifted away. Such a process continues until the end of reheating, resulting in a continuum graviton spectrum at the present universe. The graviton spectrum is calculated as

$$
\begin{aligned}
\rho_{h, k}\left(t_{0}\right) & =\mathcal{C} \times \rho_{\phi}\left(t_{k}\right) \frac{\Gamma^{\text {(grav) }}(\phi \phi \rightarrow h h)}{H_{k}}\left(\frac{a\left(t_{k}\right)}{a_{0}}\right)^{4} \\
& \simeq \begin{cases}\frac{3 \mathcal{C}}{64 \pi} m_{\phi} H_{\text {end }}^{3}\left(\frac{m_{\phi} a_{\text {end }}}{k}\right)^{1 / 2}\left(\frac{a_{\text {end }}}{a_{0}}\right)^{4} & \text { for } k \lesssim k_{\text {high }}, \\
\frac{3 \mathcal{C}}{64 \pi} m_{\phi} H_{\text {end }}^{3}\left(\frac{m_{\phi} a_{\text {end }}}{k_{\text {high }}}\right)^{1 / 2}\left(\frac{a_{\text {end }}}{a_{0}}\right)^{4} e^{-2 k^{2} / k_{\text {high }}^{2}} & \text { for } k \gtrsim k_{\text {high }},\end{cases}
\end{aligned}
$$

where $\mathcal{C}$ is an $\mathcal{O}(1)$ coefficient and $t_{k}$ is defined through $k=a\left(t_{k}\right) m_{\phi}$, i.e., the cosmic time at which the present graviton frequency $k / a_{0}$ was emitted. Here $k_{\text {high }}$ is defined as $k_{\text {high }}=\mathcal{C}_{k} m_{\phi} a\left(H=\Gamma_{\phi}\right)$ with $\mathcal{C}_{k}$ being an $\mathcal{O}(1)$ coefficient. The analytic estimate (19) with

\#3 Ref. [28] calculated the gravitational production rate analytically including $\mathcal{O}(1)$ numerical factor for a scalar particle. The same result is applied for a graviton production, since the graviton action is the same as the minimal massless scalar. 
$\mathcal{C}=3$ and $\mathcal{C}_{k}=1.5$ is also plotted in Fig. 1 and it agrees well with the numerical result. We used the function $g$ in Eq. (22) to interpolate between $k \lesssim k_{\text {high }}$ and $k \gtrsim k_{\text {high }}$. Note that there is a small deviation around $k \sim a_{\text {end }} H_{\text {end }}$. This is because there is an intermediate epoch around the end of inflation, in which the inflaton oscillation may not be regarded as a harmonic oscillation, while the analytic estimate (19) assumes the harmonic inflaton oscillation. For low scale inflation models with a large hierarchy between $m_{\phi}$ and $H_{\text {inf }}$, it is more difficult to treat this intermediate epoch, but the high frequency behavior $k \gtrsim a_{\text {end }} m_{\phi}$ is expected to be well described by the above picture.

In the above picture, the typical momentum of gravitons being produced is constant in time and is around the inflaton mass $m_{\phi}$. Therefore, it is crucial to use the inflaton equation of motion (15) to get the correct result, since otherwise the scale $m_{\phi}$ never appears in the system. In order to stress this point, in Fig. 1, we also show as the dashed lines the graviton spectrum computed assuming a smooth background evolution

$$
\rho_{\phi}=\frac{\rho_{\phi, \mathrm{inf}}}{1+\left(a / a_{\mathrm{end}}\right)^{3} e^{\Gamma_{\phi}\left(t-t_{\mathrm{end}}\right)}} \simeq \begin{cases}\rho_{\phi, \mathrm{inf}} & \text { for } t<t_{\mathrm{end}}, \\ \rho_{\phi, \mathrm{inf}}\left(\frac{a}{a_{\mathrm{end}}}\right)^{-3} e^{-\Gamma_{\phi}\left(t-t_{\mathrm{end}}\right)} & \text { for } t>t_{\mathrm{end}}\end{cases}
$$

together with Eqs. (14) and (16), which do not have the timescale $m_{\phi}$. One can clearly see that the resultant graviton spectrum is highly suppressed in high frequencies compared to the solid lines. Thus, the inflaton oscillation is crucial for the high-frequency behavior of the spectrum.

\section{Stochastic gravitational wave background revisited}

Now we plot the present stochastic GW background spectrum in terms of $\Omega_{\mathrm{GW}}(k) \equiv \rho_{h, k} / \rho_{\mathrm{cr}}$. First, the energy spectrum of subhorizon modes induced by the inflaton oscillation is given by Eq. (19) and hence

$$
\Omega_{\mathrm{GW}}^{\text {(osc) }}(k) \simeq 6 \times 10^{-24}\left(\frac{m_{\phi}}{10^{13} \mathrm{GeV}}\right)\left(\frac{H_{\mathrm{end}}}{10^{13} \mathrm{GeV}}\right)^{1 / 3}\left(\frac{T_{\mathrm{R}}}{10^{10} \mathrm{GeV}}\right)^{4 / 3}\left(\frac{a_{\mathrm{end}} m_{\phi}}{k}\right)^{1 / 2} g(k),
$$

where we used $\mathcal{C} \simeq 3$ and

$$
g(k) \simeq\left[1+\left(\frac{k}{k_{\text {high }}}\right)^{1 / 2}\right] e^{-2 k^{2} / k_{\text {high }}^{2}}
$$

The low and high frequency end of the spectrum are given respectively as

$$
f_{\text {low }}=\frac{m_{\phi}}{2 \pi} \frac{a_{\text {end }}}{a_{0}} \simeq 1.1 \times 10^{6} \mathrm{~Hz}\left(\frac{m_{\phi}}{10^{13} \mathrm{GeV}}\right)\left(\frac{T_{\mathrm{R}}}{10^{10} \mathrm{GeV}}\right)^{1 / 3}\left(\frac{10^{13} \mathrm{GeV}}{H_{\text {end }}}\right)^{2 / 3},
$$


and

$$
f_{\text {high }}=\frac{k_{\text {high }}}{2 \pi a_{0}} \simeq 2.9 \times 10^{13} \mathrm{~Hz}\left(\frac{m_{\phi}}{10^{13} \mathrm{GeV}}\right)\left(\frac{10^{10} \mathrm{GeV}}{T_{\mathrm{R}}}\right),
$$

where we used $\mathcal{C}_{k} \simeq 1.5$. For $f>f_{\text {high }}$ the spectrum decays exponentially. Remember that the present frequency $f$ is related to the comoving wavenumber $k$ through $f=k /\left(2 \pi a_{0}\right)$.

On the other hand, there are also contributions from the superhorizon modes that exit the horizon during inflation and reenter the horizon after inflation [32-34]. The shape of present GW spectrum depends on the equation of state of the Universe. In particular, the GW spectrum scales as $\Omega_{\mathrm{GW}} \propto k^{0}\left(k^{-2}\right)$ for modes that enter the horizon during radiation (matter) domination [35-37]. The GW spectrum is evaluated as

$$
\Omega_{\mathrm{GW}}^{(\mathrm{inf})}(k) \simeq \Omega_{m}^{2} \frac{3 r}{128} \mathcal{P}_{\zeta}\left(k_{0}\right)\left(\frac{k_{0}}{k}\right)^{2-n_{t}}\left(\frac{g_{*}\left(T_{k}\right)}{g_{*}\left(T_{\mathrm{eq}}\right)}\right)\left(\frac{g_{* s}\left(T_{\mathrm{eq}}\right)}{g_{* s}\left(T_{k}\right)}\right)^{4 / 3} T_{1}\left(\frac{k}{k_{\mathrm{eq}}}\right) T_{2}\left(\frac{k}{k_{\mathrm{R}}}\right),
$$

where $k_{0} / a_{0}=H_{0}$ is the Hubble parameter at present, $r$ is the tensor-to-scalar ratio, $n_{t}=$ $-r / 8$ is the tensor spectral index, $T_{1}(x) \simeq 1+(32 / 9) x^{2}$ and $T_{2}(x) \simeq\left(1+x^{2}\right)^{-1}$, and

$$
f_{\mathrm{R}}=\frac{H_{\mathrm{R}}}{2 \pi} \frac{a_{\mathrm{R}}}{a_{0}} \simeq 2.6 \times 10^{2} \mathrm{~Hz}\left(\frac{g_{* s}\left(T_{\mathrm{R}}\right)}{106.75}\right)^{1 / 6}\left(\frac{T_{\mathrm{R}}}{10^{10} \mathrm{GeV}}\right) .
$$

This GW spectrum is cut at the frequency $f_{\text {end }}$ :

$$
f_{\text {end }}=\frac{H_{\text {end }}}{2 \pi} \frac{a_{\text {end }}}{a_{0}} \simeq 1.1 \times 10^{6} \mathrm{~Hz}\left(\frac{H_{\text {end }}}{10^{13} \mathrm{GeV}}\right)^{1 / 3}\left(\frac{T_{\mathrm{R}}}{10^{10} \mathrm{GeV}}\right)^{1 / 3} .
$$

Fig. 2 shows the stochastic GW background spectrum for $H_{\text {inf }}=10^{14} \mathrm{GeV}, H_{\text {end }}=m_{\phi}=$ $10^{13} \mathrm{GeV}$ and $T_{\mathrm{R}}=10^{12} \mathrm{GeV}$ (left) and $10^{10} \mathrm{GeV}$ (right). The solid lines correspond to the vacuum contribution that is amplified due to the inflaton oscillation during the reheating era and the dashed lines correspond to the inflationary GW that is amplified during the inflation stage.

\section{Discussion}

We have shown that there is inevitably a contribution to the stochastic GW background from the reheating era. It is the subhorizon graviton excitation amplified by the inflaton oscillation. As shown in Fig. 2, this extends to the high-frequency tail which scales as $\Omega_{\mathrm{GW}} \propto f^{-1 / 2}$ in addition to the well-known inflationary GWs that exit the horizon during inflation and reenter the horizon after inflation. This high frequency tail contains a lot of information about the property of the inflaton: the inflaton mass, the inflaton lifetime (or the reheating temperature) and so on. Although we have focused on the simple quadratic inflaton potential, it is expected that the high frequency tail exhibits more nontrivial structure for a 

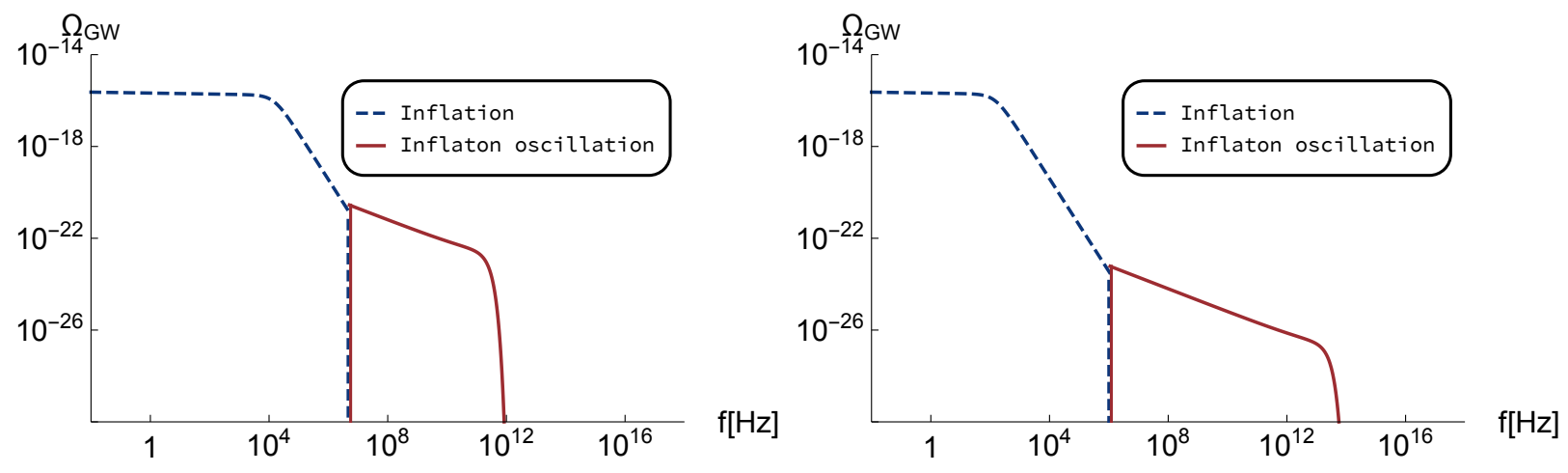

Figure 2: Stochastic GW background spectrum for $H_{\text {inf }}=10^{14} \mathrm{GeV}, H_{\text {end }}=m_{\phi}=10^{13} \mathrm{GeV}$, and $T_{\mathrm{R}}=10^{12} \mathrm{GeV}$ (left) and $10^{10} \mathrm{GeV}$ (right). The solid lines correspond to the vacuum contribution that is amplified due to the inflaton oscillation during the reheating era and the dashed lines correspond to the inflationary GW that is amplified during the inflation stage.

more general form of the inflaton potential. We will come back to this issue in a separate work.

Lastly we discuss other contributions to the high-frequency stochastic GW background spectrum, which can hide the vacuum contributions that we found. The Standard Model thermal plasma emits gravitons through scattering processes and they also constitute a stochastic GW background [22,23]. The typical frequency of the emitted graviton at the temperature $T$ is of order $T$ and it is redshifted as $a^{-1}(t)$. Since the temperature is also redshifted as $a^{-1}(t)$, the typical comoving frequency (or the frequency observed today) is roughly the same independently of the temperature. The overall amount of GW is dominated by those emitted earlier epoch for all the frequency range, i.e., at the highest temperature $T_{\mathrm{R}}$ and the result is $\# 4$

$$
\Omega_{\mathrm{GW}}^{(\mathrm{th})}(k) \sim 2 \times 10^{-13}\left(\frac{T_{\mathrm{R}}}{10^{10} \mathrm{GeV}}\right)\left(\frac{k}{a_{*} T_{*}}\right)^{3} \varphi\left(\frac{k}{a_{*} T_{*}}\right)
$$

where $T_{*}$ denotes the reference temperature taken to be the electroweak scale, and $\varphi(x) \simeq 1$ for $x \ll 1$ and exponentially decreases for $x \gtrsim 1$. Another contribution comes from the graviton bremsstrahlung processes associated with the perturbative inflaton decay. The spectrum is given by $[24,25]$

$$
\Omega_{\mathrm{GW}}^{(\mathrm{brem})}(k) \simeq \Omega_{r} \frac{m_{\phi}^{2}}{16 \pi^{2} M_{P}^{2}} \frac{f}{f_{\text {high }}},
$$

for $f \ll f_{\text {high }}$. Note that the coupling that is responsible for the inflaton decay may also induce the preheating and resonant particle production if the coupling is relatively large [38-

\footnotetext{
\#4 The dilute plasma before the completion of the reheating also emit gravitons. However, one can show that this contribution goes like $k^{4.6}$ toward lower frequency and is hidden by the $k^{3}$ tail of (28).
} 
42]. It may act as a classical source of GWs resulting in more abundant GW background than Eq. (29) [4], but it is rather model dependent and we do not go into details here. Although in the most parameter regions these contributions are larger than those from the inflaton oscillation, it might be possible to remove these contributions from the data and find the inflaton oscillation signal, which will provide us with rich information on the early universe and the nature of the inflaton. We emphasize that the high frequency GWs induced by the inflaton oscillation may not be regarded as classical waves since they never exit the horizon and the occupation number is much smaller than unity. Thus detection of such high frequency GWs may be regarded as a direct test of quantum nature of the graviton.

\section{Acknowledgments}

This work was supported by the Grant-in-Aid for Scientific Research C (No.18K03609 [KN]) and Innovative Areas (No.17H06359 [KN]). The work of RJ is supported by Grants-inAid for JSPS Overseas Research Fellow (No. 201960698). This work was supported by the Deutsche Forschungsgemeinschaft under Germany's Excellence Strategy - EXC 2121 "Quantum Universe" - 390833306. This work was also supported by the ERC Starting Grant "NewAve" (638528).

\section{References}

[1] A. A. Starobinsky, "Spectrum of relict gravitational radiation and the early state of the universe," JETP Lett. 30 (1979) 682-685. [Pisma Zh. Eksp. Teor. Fiz.30,719(1979)].

[2] M. Maggiore, Gravitational Waves. Vol. 2: Astrophysics and Cosmology. Oxford University Press, 3, 2018.

[3] M. Giovannini, "Primordial backgrounds of relic gravitons," Prog. Part. Nucl. Phys. 112 (2020) 103774, arXiv:1912.07065 [astro-ph.CO].

[4] S. Y. Khlebnikov and I. I. Tkachev, "Relic gravitational waves produced after preheating," Phys. Rev. D56 (1997) 653-660, arXiv:hep-ph/9701423 [hep-ph].

[5] A. Vilenkin and E. P. S. Shellard, Cosmic Strings and Other Topological Defects. Cambridge University Press, 2000. http://www. cambridge.org/mw/academic/subjects/physics/ theoretical-physics-and-mathematical-physics/ cosmic-strings-and-other-topological-defects?format=PB.

[6] E. Witten, "Cosmic Separation of Phases," Phys. Rev. D30 (1984) 272-285.

[7] C. J. Hogan, "Gravitational radiation from cosmological phase transitions," Mon. Not. Roy. Astron. Soc. 218 (1986) 629-636.

[8] C. Caprini and D. G. Figueroa, "Cosmological Backgrounds of Gravitational Waves," Class. Quant. Grav. 35 no. 16, (2018) 163001, arXiv:1801.04268 [astro-ph.C0]. 
[9] Y. Ema, R. Jinno, K. Mukaida, and K. Nakayama, "Gravitational Effects on Inflaton Decay," JCAP 1505 (2015) 038, arXiv:1502.02475 [hep-ph].

[10] S. Kawamura et al., "The Japanese space gravitational wave antenna DECIGO," Class. Quant. Grav. 23 (2006) S125-S132.

[11] M. Punturo et al., "The Einstein Telescope: A third-generation gravitational wave observatory," Class. Quant. Grav. 27 (2010) 194002.

[12] G. Janssen et al., "Gravitational wave astronomy with the SKA," PoS AASKA14 (2015) 037, arXiv:1501.00127 [astro-ph.IM].

[13] LIGO Scientific, Virgo Collaboration, B. P. Abbott et al., "Upper Limits on the Stochastic Gravitational-Wave Background from Advanced LIGO's First Observing Run," Phys. Rev. Lett. 118 no. 12, (2017) 121101, arXiv:1612.02029 [gr-qc]. [Erratum: Phys.Rev.Lett. 119, 029901 (2017)].

[14] LISA Collaboration, P. Amaro-Seoane et al., "Laser Interferometer Space Antenna," arXiv:1702.00786 [astro-ph.IM].

[15] MAGIS Collaboration, P. W. Graham, J. M. Hogan, M. A. Kasevich, S. Rajendran, and R. W. Romani, "Mid-band gravitational wave detection with precision atomic sensors," arXiv:1711.02225 [astro-ph.IM].

[16] AEDGE Collaboration, Y. A. El-Neaj et al., "AEDGE: Atomic Experiment for Dark Matter and Gravity Exploration in Space," EPJ Quant. Technol. 7 (2020) 6, arXiv: 1908.00802 [gr-qc].

[17] F. Li, J. Baker, Robert M.L., Z. Fang, G. V. Stephenson, and Z. Chen, "Perturbative Photon Fluxes Generated by High-Frequency Gravitational Waves and Their Physical Effects," Eur. Phys. J. C 56 (2008) 407-423, arXiv:0806.1989 [gr-qc].

[18] F. Li, N. Yang, Z. Fang, J. Baker, Robert M.L., G. V. Stephenson, and H. Wen, "Signal Photon Flux and Background Noise in a Coupling Electromagnetic Detecting System for High Frequency Gravitational Waves," Phys. Rev. D 80 (2009) 064013, arXiv:0909.4118 [gr-qc].

[19] A. Ejlli, D. Ejlli, A. M. Cruise, G. Pisano, and H. Grote, "Upper limits on the amplitude of ultra-high-frequency gravitational waves from graviton to photon conversion," Eur. Phys. J. C 79 no. 12, (2019) 1032, arXiv: 1908.00232 [gr-qc].

[20] A. Ito, T. Ikeda, K. Miuchi, and J. Soda, "Probing GHz gravitational waves with graviton-magnon resonance," Eur. Phys. J. C 80 no. 3, (2020) 179, arXiv:1903.04843 [gr-qc].

[21] A. Ito and J. Soda, "A formalism for magnon gravitational wave detectors," arXiv:2004.04646 [gr-qc].

[22] J. Ghiglieri and M. Laine, "Gravitational wave background from Standard Model physics: Qualitative features," JCAP 1507 (2015) 022, arXiv:1504.02569 [hep-ph]. 
[23] J. Ghiglieri, G. Jackson, M. Laine, and Y. Zhu, "Gravitational wave background from Standard Model physics: Complete leading order," arXiv:2004.11392 [hep-ph].

[24] K. Nakayama and Y. Tang, "Stochastic Gravitational Waves from Particle Origin," Phys. Lett. B788 (2019) 341-346, arXiv:1810.04975 [hep-ph].

[25] D. Huang and L. Yin, "Stochastic Gravitational Waves from Inflaton Decays," Phys. Rev. D100 no. 4, (2019) 043538, arXiv:1905.08510 [hep-ph].

[26] Y. Ema, R. Jinno, K. Mukaida, and K. Nakayama, "Gravitational particle production in oscillating backgrounds and its cosmological implications," Phys. Rev. D94 no. 6, (2016) 063517, arXiv:1604.08898 [hep-ph].

[27] Y. Ema, K. Nakayama, and Y. Tang, "Production of Purely Gravitational Dark Matter," JHEP 09 (2018) 135, arXiv:1804.07471 [hep-ph].

[28] D. J. H. Chung, E. W. Kolb, and A. J. Long, "Gravitational production of super-Hubble-mass particles: an analytic approach," JHEP 01 (2019) 189, arXiv:1812.00211 [hep-ph].

[29] A. D. Linde, "Chaotic Inflation," Phys. Lett. B 129 (1983) 177-181.

[30] C. Destri, H. J. de Vega, and N. Sanchez, "MCMC analysis of WMAP3 and SDSS data points to broken symmetry inflaton potentials and provides a lower bound on the tensor to scalar ratio," Phys. Rev. D 77 (2008) 043509, arXiv: astro-ph/0703417.

[31] K. Nakayama, F. Takahashi, and T. T. Yanagida, "Polynomial Chaotic Inflation in the Planck Era," Phys. Lett. B $\mathbf{7 2 5}$ (2013) 111-114, arXiv:1303.7315 [hep-ph].

[32] M. Maggiore, "Gravitational wave experiments and early universe cosmology," Phys. Rept. 331 (2000) 283-367, arXiv:gr-qc/9909001 [gr-qc].

[33] T. L. Smith, M. Kamionkowski, and A. Cooray, "Direct detection of the inflationary gravitational wave background," Phys. Rev. D73 (2006) 023504, arXiv:astro-ph/0506422 [astro-ph].

[34] L. A. Boyle and P. J. Steinhardt, "Probing the early universe with inflationary gravitational waves," Phys. Rev. D77 (2008) 063504, arXiv:astro-ph/0512014 [astro-ph].

[35] K. Nakayama, S. Saito, Y. Suwa, and J. Yokoyama, "Space laser interferometers can determine the thermal history of the early Universe," Phys. Rev. D77 (2008) 124001, arXiv:0802.2452 [hep-ph].

[36] K. Nakayama, S. Saito, Y. Suwa, and J. Yokoyama, "Probing reheating temperature of the universe with gravitational wave background," JCAP 0806 (2008) 020, arXiv:0804.1827 [astro-ph].

[37] S. Kuroyanagi, T. Chiba, and N. Sugiyama, "Precision calculations of the gravitational wave background spectrum from inflation," Phys. Rev. D79 (2009) 103501, arXiv:0804. 3249 [astro-ph] . 
[38] A. Dolgov and D. Kirilova, "ON PARTICLE CREATION BY A TIME DEPENDENT SCALAR FIELD," Sov. J. Nucl. Phys. 51 (1990) 172-177.

[39] J. H. Traschen and R. H. Brandenberger, "Particle Production During Out-of-equilibrium Phase Transitions," Phys. Rev. D 42 (1990) 2491-2504.

[40] L. Kofman, A. D. Linde, and A. A. Starobinsky, "Reheating after inflation," Phys. Rev. Lett. 73 (1994) 3195-3198, arXiv:hep-th/9405187.

[41] Y. Shtanov, J. H. Traschen, and R. H. Brandenberger, "Universe reheating after inflation," Phys. Rev. D 51 (1995) 5438-5455, arXiv:hep-ph/9407247.

[42] L. Kofman, A. D. Linde, and A. A. Starobinsky, "Towards the theory of reheating after inflation," Phys. Rev. D 56 (1997) 3258-3295, arXiv:hep-ph/9704452. 